\title{
The Performance of H263-Based Video Telephony Over Turbo-Equalized GSM/GPRS
}

\author{
Peter Cherriman, Bee Leong Yeap, and Lajos Hanzo
}

\begin{abstract}
The performance of turbo-equalized Global System of Mobile Communications/General Packet Radio Service (GSM/GPRS)-like videophone transceivers is studied over dispersive fading channels as a function of the number of turbo equalization iterations. Iteration gains up to $6 \mathrm{~dB}$ were attained. The operating channel signal-to-noise ratio (SNR) required for maintaining a peak SNR loss of less than $1 \mathrm{~dB}$ was reduced by 3.2 dB for the one-slot system and by $4.1 \mathrm{~dB}$ for the four-slot system. The proposed system is capable of providing low-resolution videophone services over GSM/GPRS.
\end{abstract}

Index Terms-GSM, GPRS, turbo equalization, wireless video telephone.

\section{MOTIVATION AND BACKGROUND}

$\mathbf{T}$ HE EXISTING second-generation (2G) wireless systems [1] now constitute a mature technology. In the context of these $2 \mathrm{G}$ systems, attractive value-added services can be offered to a plethora of existing users with the advent of video telephony. Although the $2 \mathrm{G}$ systems have not been designed with video communications in mind, with the aid of the specially designed error-resilient, fixed-rate video codecs [3]-[6] proposed by Streit $e t$ al., it is nonetheless realistic to provide videophone services over these low-rate schemes. In our previous work, we designed a suite of fixed-rate, proprietary video codecs [3]-[6] that are capable of operating at a video scanning or refreshment rate of 10 frames/s over an additional speech channel of the $2 \mathrm{G}$ systems. More explicitly, these video codecs were capable of maintaining sufficiently low bit rates for the provision of videophony over an additional speech channel in the context of the operational $2 \mathrm{G}$ wireless systems [1], provided that low-dynamic head-and-shoulder video-sequences of the $176 \times 144$-pixel so-called quarter common intermediate format (QCIF) or $128 \times 96$-pixel sub-QCIF video resolution are employed. We note, however, that for high-dynamic sequences, the $32 \mathrm{~kb} / \mathrm{s}$ typical speech bit rate of the cordless telephone systems [1], such as the Japanese PHS, the digital European cordless telephone (DECT), or the British CT2 system, is more adequate in terms of video quality. Furthermore, the proposed programmable video codecs are capable of multirate operation in the third-generation $(3 \mathrm{G})$ universal mobile telecommunications system (UMTS) [2], [1] or in the so-called IMT2000 [2], [1] and cdma2000 [2], [1] systems.

While in [3]-[7], constant video rate proprietary video codecs and reconfigurable quadrature amplitude modulation

Manuscript received April 1, 2000; revised November 12, 2001. This work was supported by the Mobile Virtual Centre of Excellence, U.K. This paper was recommended by Associate Editor O. K. Al-Shaykh.

The authors are with the Department of Electronic and Computer Science, University of Southampton, Southampton SO17 1BJ, U.K. (e-mail lh@ecs.soton.ac.uk).

Digital Object Identifier 10.1109/TCSVT.2002.804898
TABLE I

SYSTEM PARAMETERS

\begin{tabular}{l|l}
\hline \multicolumn{2}{c}{ Simulation Parameters } \\
\hline \hline Channel Model & COST-207 Hilly Terrain \\
Carrier Frequency & $900 \mathrm{MHz}$ \\
Vehicular Speed & $30 \mathrm{mph}$ \\
Doppler Frequency & $40.3 \mathrm{~Hz}$ \\
Modulation & GMSK, $B_{n}=0.3$ \\
Channel Coding & Convol. $(\mathrm{n}, \mathrm{k}, \mathrm{K})=(2,1,5)$ \\
Octal Generator Polynomials & 23,33 \\
Channel Interleavers & Random $(232,928)$ \\
Max Turbo Equalisation Iterations & 10 \\
No. of TDMA Frame per Packet & 2 \\
No. of Slots per TDMA Frame & 1,4 \\
Convolutional Decoder Algorithm & Log-MAP \\
Equaliser Algorithm & Log-MAP \\
\hline
\end{tabular}

(QAM) based [8] transceivers were used, in this contribution, constant-envelope Gaussian minimum shift keying (GMSK) [1] was advocated. Specifically, the feasibility of H.263-based video telephony was investigated in the context of an enhanced turbo-equalized GSM-like system, which can rely on power-efficient class-C amplification. The associated speech compression and transmission aspects are beyond the scope of this contribution [9].

The outline of the paper is as follows. Section II summarizes the associated system parameters and system's schematic, while Section III provides a brief overview of turbo equalization. Section IV characterizes the system both in terms of turbo equalization performance and video performance. Lastly, Section $\mathrm{V}$ provides our conclusions. Let us now consider the outline of the system.

\section{SYSTEM PARAMETERS}

In this contribution, a Global System of Mobile Communications /General Packet Radio Service (GSM/GPRS)-based [1] videophone scheme is proposed and characterized. The associated system parameters are summarized in Table I, while the system's schematic is portrayed in Fig. 1. An advanced feature of the system is that it employs joint channel equalization and channel decoding, which is referred to as turbo equalization [10], [11]. The fundamental principles of turbo equalization are described in Section III.

The system uses the GSM frame structure [1], and the COST-207 Hilly Terrain channel model, whose impulse response is shown in Fig. 2. Each transmitted packet is interleaved over two GSM TDMA frames, in order to disperse bursty errors.

The GPRS system allows the employment of multiple timeslots per user. We studied both a GSM-like system using one slot per TDMA frame, and a GPRS-like arrangement with four slots per TDMA frame, a scenario where the user is assigned half the 


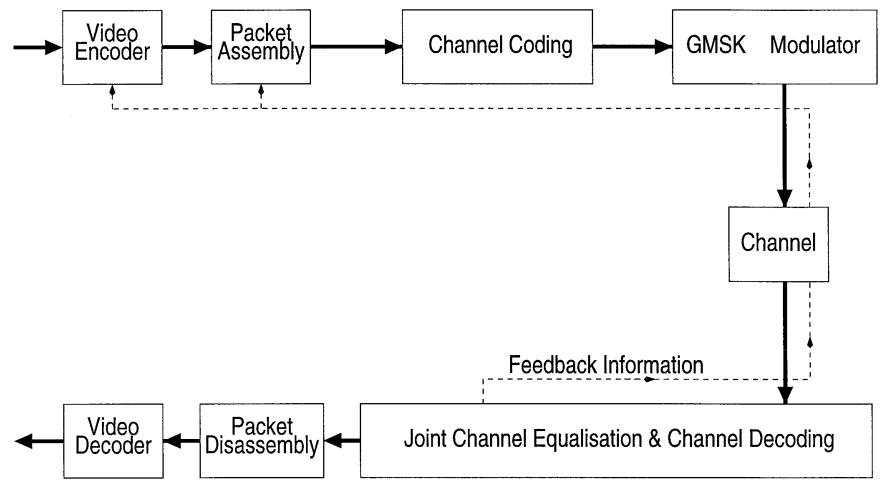

Fig. 1. System schematic for turbo-equalized video system.

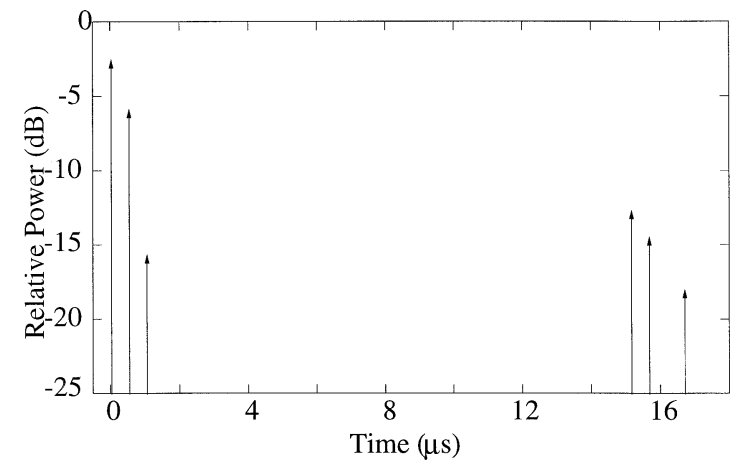

Fig. 2. COST207 Hilly Terrain channel model.

maximum capacity of an eight-slot GPRS/GSM carrier. The bit rates associated with one and four slots per TDMA frame are shown in Table II.

Assuming that the processing delay is negligible, the delay of the system is dependent on the length of the channel interleaver. As specified in Table I, the interleaver lengths of the one- and four-slot systems are 232 and 928 bits, respectively. This corresponds to the transmission of two TDMA frames, each having a duration of $4.615 \mathrm{~ms}$. Therefore, the total delay of the system is $9.23 \mathrm{~ms}$.

The effective video bitrates that can be obtained in conjunction with half-rate convolutional coding are 10 and 47.5 $\mathrm{Kb} / \mathrm{s}$ for the one and four slots per TDMA frame scenarios, respectively. Again, the system's schematic is shown in Fig. 1. The basic prerequisite for the duplex video system's operation is that-as seen in Fig. 1-the channel decoder's output is used for assessing, whether the received packet contains any transmission errors. Since the H.263 video codec [3] extensively employs variable-length compression techniques, it achieves a high compression ratio. However, as with all entropy- and variable-length coded bit streams, its bits are extremely sensitive to transmission errors. This error sensitivity was mitigated in our system by employing an adaptive packetization and packet dropping regime [3] for improving the error resilience of the baseline H.263 codec, which refrained from using the negotiable advanced coding options of the standard. If there were channel decoding errors, the receiver instructed the remote transmitter-by superimposing a strongly protected repetition-coded packet acknowledgment flag on to the reverse-direction message- to drop the corresponding
TABLE II

SUMMARY OF SYSTEM-SPECIFIC BIT RATES

\begin{tabular}{l|r|r}
\hline \multicolumn{3}{c}{ Bit rates etc } \\
\hline Slots/TDMA frame & 1 & 4 \\
\hline \hline Coded Bits/TDMA slot & 116 & 116 \\
Data Bits/TDMA slot & 58 & 58 \\
Data Bits/TDMA frame & 58 & 232 \\
TDMA frame/packet & 2 & 2 \\
Data Bits/packet & 116 & 464 \\
Packet Header (bits) & 8 & 10 \\
CRC (bits) & 16 & 16 \\
Video Bits/packet & 92 & 438 \\
TDMA frame length & $4.615 \mathrm{~ms}$ & $4.615 \mathrm{~ms}$ \\
TDMA frames/s & 216.68 & 216.68 \\
Video Packets per sec & 108.34 & 108.34 \\
Video bitrate (kbps) & 10.0 & 47.5 \\
Video framerate (fps) & 10 & 10 \\
\hline
\end{tabular}

video packet following the philosophy of [3] and [7]. This prevents the local and remote video reconstructed frame buffers from being contaminated by channel errors. Let us now briefly consider the associated aspects of turbo equalization. Due to these measures, the associated video performance degradation was found perceptually unobjectionable for packet dropping or transmission frame error rates (FER) below about 5\%, although this issue will be detailed in more depth during our further discourse. Due to lack of space, some of the implementation details are omitted, which can be found in [3] and [7].

\section{OVERVIEW OF TURBo EQUALIZATION}

Turbo equalization was proposed by Douillard et al. in 1995 [10], [11] for a serially concatenated rate $R=1 / 2$ convolutional-coded binary phase shift keying (BPSK) system. Specifically, Douillard et al. demonstrated that the turbo equalizer was capable of mitigating the effects of inter-symbol interference (ISI), provided that the channel impulse response (CIR) is known. Instead of performing the equalization and error-correction decoding independently, better performance can be achieved by performing the channel equalization and channel decoding jointly and iteratively. Gertsman and Lodge [12] then showed that the iterative process of turbo equalizers can compensate for the degradations due to imperfect channel estimation. Turbo equalization schemes for the Global System of Mobile Communications (GSM) were also investigated by Bauch and Franz [14], [15], and Jordan as well as Kammeyer [16], [17].

The basic philosophy of the original turbo equalization technique stems from the iterative turbo decoding algorithm consisting of two soft-in/soft-out (SISO) decoders, a structure, which was proposed by Berrou et al. [18], [19], [11]. Before proceeding with our in-depth discussion, let us briefly define below the terms a priori, a posteriori, and extrinsic information, which we employ throughout this paper.

- A priori: The a priori information associated with a bit $v_{m}$ is the information known before equalization or decoding commences, from a source other than the received sequence or the code constraints.

- Extrinsic: The extrinsic information associated with a bit $v_{m}$ is the information provided by the equalizer or decoder based 


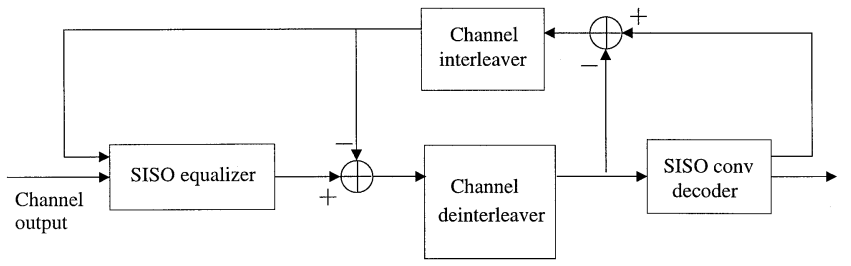

Fig. 3. Structure of original turbo equalizer introduced by Douillard et al. [10].

on the received sequence and on the a priori information of all bits with the exception of the received and a priori information explicitly related to that particular bit $v_{m}$.

- A posteriori: The a posteriori information associated with a bit is the reliability information that the SISO algorithm provides taking into account all available sources of information about the bit $u_{k}$.

As mentioned previously, the turbo equalizer of Fig. 3 consists of a SISO equalizer and a SISO decoder. The SISO equalizer in Fig. 3 generates the a posteriori probability upon receiving the corrupted transmitted signal sequence and the a priori probability provided by the SISO decoder. However, during the initial iteration stages, i.e., at the first turbo equalization iteration, no a priori information is supplied by the channel decoder. Therefore, the a priori probability is set to $1 / 2$, since the transmitted bits are assumed to be equiprobable. Before passing the a posteriori information generated by the SISO equalizer to the SISO decoder of Fig. 3, the contribution of the decoder, which is in the form of the a priori information, accruing from the previous iteration must be removed, in order to yield the combined channel and extrinsic information. This also minimizes the correlation between the a priori information supplied by the decoder and the a posteriori information generated by the equalizer. The term "combined channel and extrinsic information" indicates that they are inherently linked-in fact they are typically induced by mechanisms, which exhibit memory - and hence they cannot be separated. The removal of the a priori information is necessary, in order to prevent the decoder from "reprocesing" its own information, which would result in the so-called " "positive feedback" phenomenon, overwhelming the decoder's current reliability-estimation of the coded bits, i.e., the extrinsic information.

The combined channel and extrinsic information is channeldeinterleaved and directed to the SISO decoder, as depicted in Fig. 3. Subsequently, the SISO decoder computes the a posteriori probability of the coded bits. Note that the latter steps are different from those in turbo decoding, which only produces the a posteriori probability of the source bits, rather than those of all channel coded bits. The combined deinterleaved channel and extrinsic information is then removed from the a posteriori information provided by the decoder in Fig. 3 before channel interleaving, in order to yield the extrinsic information. This is to prevent the channel equalizer from receiving information based on its own decisions, which was generated in the previous turbo equalization iteration. The extrinsic information computed is then employed as the a priori input information of the equalizer in the next channel equalization process. This constitutes the first turbo equalization iteration. The iterative process is repeated, until the required termination criteria are met [13]. At

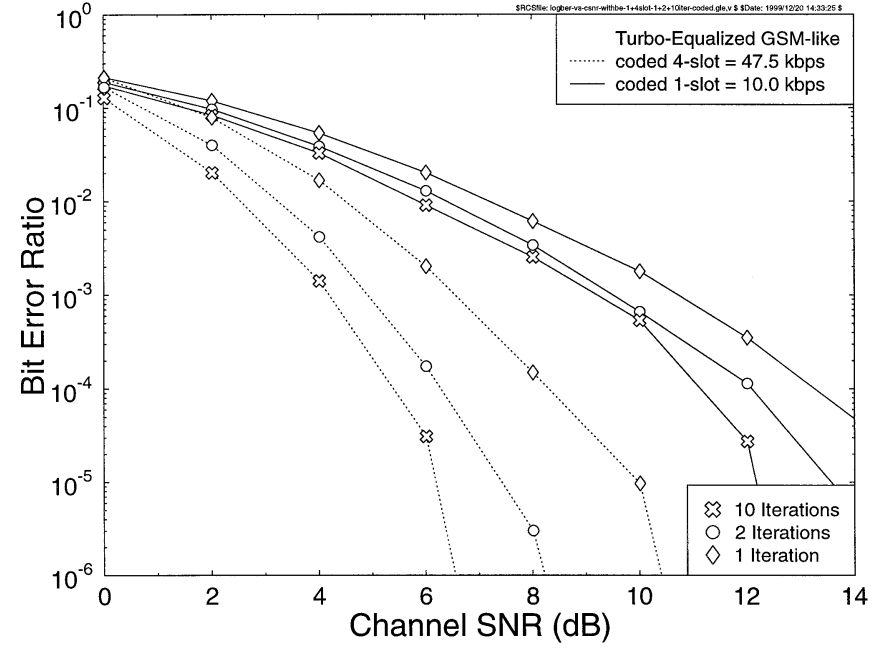

Fig. 4. BER versus channel SNR for one and four slots per TDMA frame, and for one, two, and ten turbo-equalizer iterations, over the channel of Fig. 2.

this stage, the a posteriori information of the source bits, which has been generated by the decoder is utilized to estimate the transmitted bits.

\section{Turbo Equalization Performance}

Let us now characterize the performance of our video system. Fig. 4 shows the bit-error rate (BER) versus channel signal-tonoise ratio (SNR) for the one- and four-slot scenarios after one, two, and ten iterations of the turbo equalizer. The figure shows the BER performance improvement upon each iteration of the turbo equalizer, although there is only a limited extra performance improvement after five iterations. The figure also shows that the four-slot scenario has a lower BER than the one-slot scenario. This is because the four-slot scenario has a longer interleaver, which renders the turbo equalization process more effective due to its increased time-diversity.

Let us now consider the associated packet-loss ratio (PLR) versus channel SNR performance in Fig. 5. The PLR is a more pertinent measure of the expected video performance, since — based on the philosophy of [3], [7]—our video scheme discards all video packets, which are not error-free. Hence, our goal is to maintain as low a PLR, as possible. Observe in Fig. 5 that the associated iteration gains are more pronounced in terms of the PLR than in the BER. Specifically, at BER $=10^{-3}$ and at PLR $=10^{-3}$, the iteration gain obtained by the four-slot system after performing ten turbo equalization iterations is 2 and $5 \mathrm{~dB}$, respectively.

It should be noted furthermore that for low SNRs, the packet-loss performance of the four-slot system is inferior to that of the one-slot system, while the BER is similar or better at the same SNRs. This is because the probability of having a single bit error in the four-slot video packet is higher due to its quadruple length. This phenomenon will be further augmented in Section IV-B.

\section{A. Video Performance}

The video quality performance is directly related to the PLR of our video system. Fig. 6 shows the associated average peak 


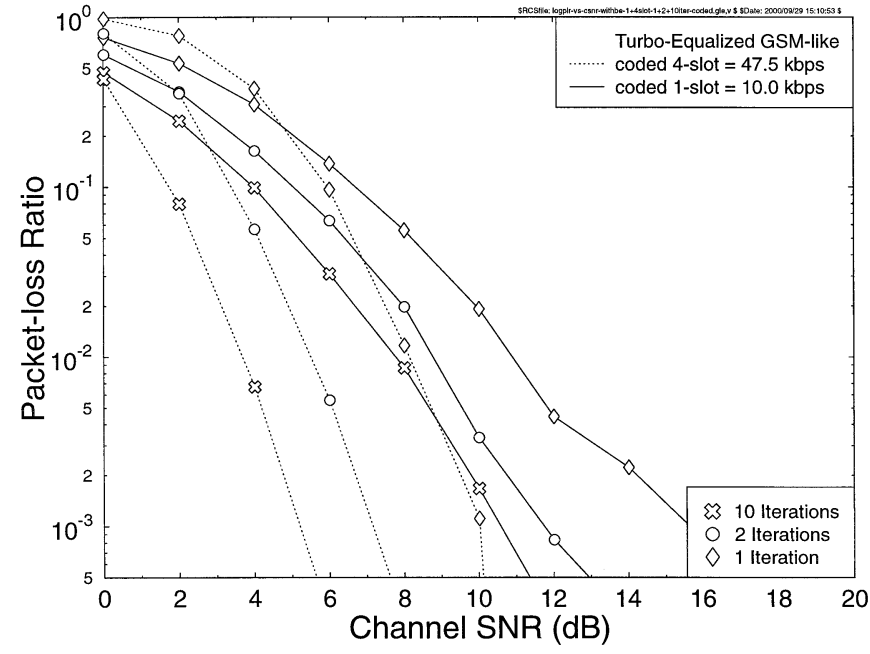

Fig. 5. Video PLR versus channel SNR for one and four slots per TDMA frame, and for one, two, and ten turbo equalizer iterations over the channel of Fig. 2 using convolutional coding.

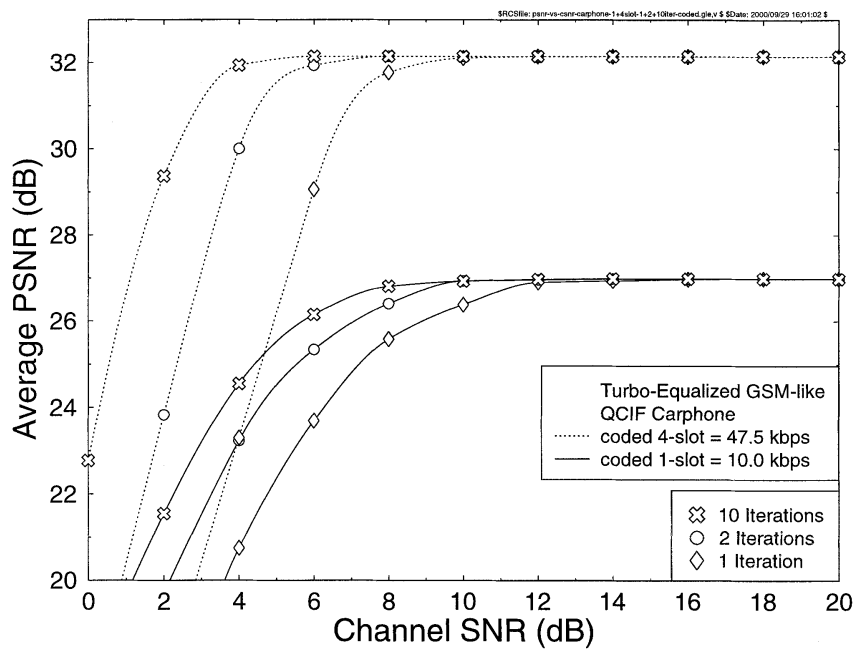

Fig. 6. Video quality in PSNR (decibels) versus channel SNR for one and four slots per TDMA frame, and for one, two, and ten iterations of the turbo equalizer upon using the highly motion active "Carphone" video sequence over the channel of Fig. 2 using convolutional coding.

SNR versus channel SNR performance, demonstrating that an improved video quality can be maintained at lower SNRs, as the number of iterations increases. Additionally, the higher bit rate of the four-slot system corresponds to a higher overall video quality. Explicitly, up to 6-dB SNR gain can be achieved after ten turbo equalization iterations, as seen in Fig. 6, while maintaining a given PSNR.

Fig. 6 characterized the performance for the highly motion-active "Carphone" sequence. However, the performance improvements are similar for the low-activity "Miss America" video sequence, as seen in Fig. 7. Observe that the lower activity "Miss America" video sequence is represented at a higher video quality at the same video bit rate. A deeper insight into the achievable video quality improvement in conjunction with turbo equalization can be provided by plotting the video quality measured in PSNR (decibels) versus time, as seen in Fig. 8, for the "Miss America" video sequence using the

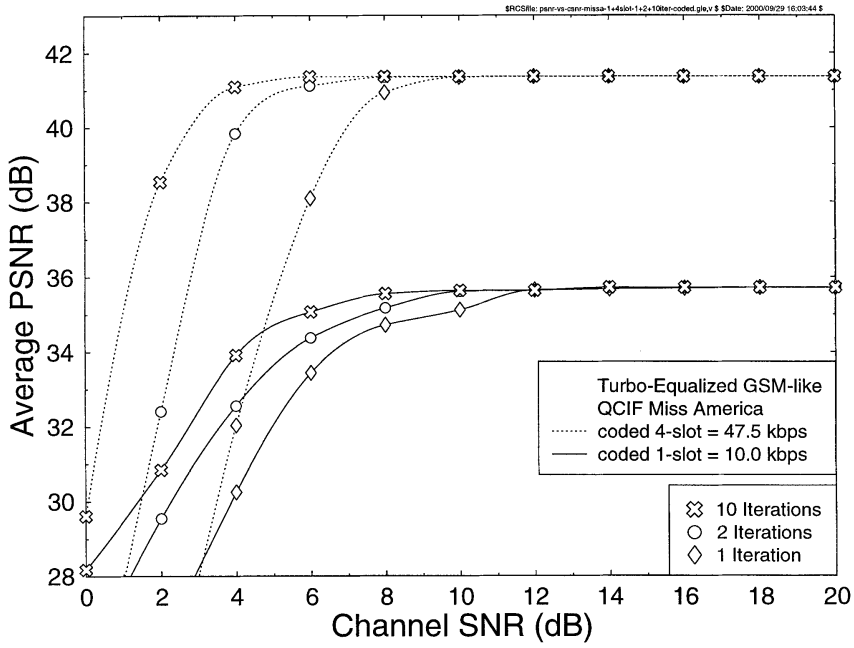

Fig. 7. Video quality in PSNR (decibels) versus channel SNR for one and four slots per TDMA frame, and for one, two, and ten turbo equalizer iterations, using the low-activity "Miss America" video sequence over the channel of Fig. 2 using convolutional coding.

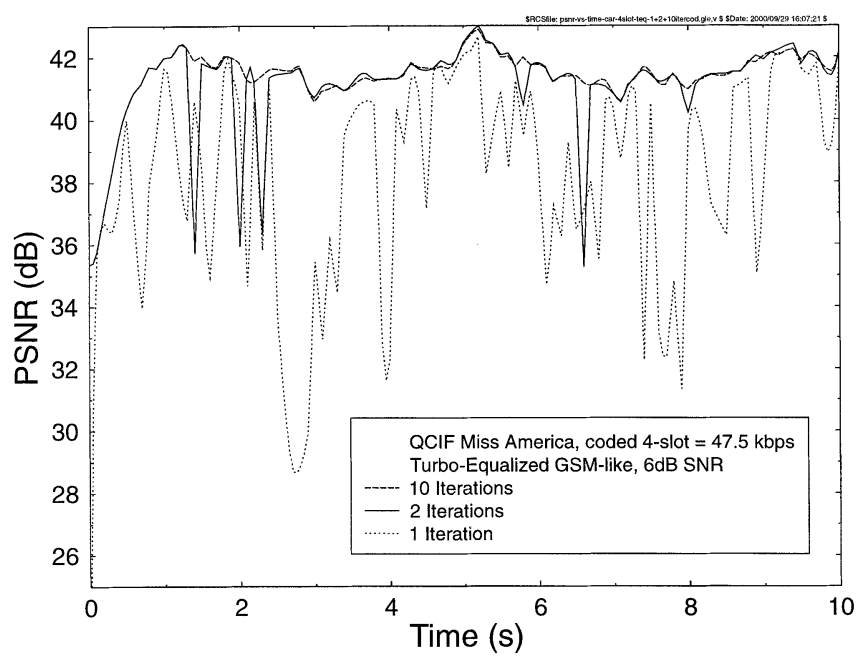

Fig. 8. Video quality in PSNR (decibels) versus time using four slots per TDMA frame, and for one, two, and ten iterations of the turbo equalizer for the low-activity "Miss America" video sequence using convolutional coding.

TABLE III

MiNIMUM REQUiRed OPERATING CHANNEL SNR FOR THE QCIF CARPHONE SEQUENCE OVER THE CHANNEL OF FIG. 2

\begin{tabular}{l|l|l}
\hline \multicolumn{3}{c}{ Channel SNR for 1 dB loss of PSNR } \\
\hline Slots/TDMA frame & 1 & 4 \\
\hline \hline 1 Iteration & $9.0 \mathrm{~dB}$ & $7.5 \mathrm{~dB}$ \\
2 Iterations & $7.2 \mathrm{~dB}$ & $5.2 \mathrm{~dB}$ \\
3 Iterations & $6.44 \mathrm{~dB}$ & $3.9 \mathrm{~dB}$ \\
4 Iterations & $6.37 \mathrm{~dB}$ & $3.7 \mathrm{~dB}$ \\
10 Iterations & $5.8 \mathrm{~dB}$ & $3.4 \mathrm{~dB}$ \\
\hline
\end{tabular}

four-slot system at a channel SNR of $6 \mathrm{~dB}$ for one, two, and ten iterations of the turbo equalizer.

Specifically, the bottom trace of the figure shows how the video quality varies in the one-iteration scenario, which is equivalent to conventional equalization. The sudden reductions in video quality are caused by packet-loss events, which result in parts of the picture being "frozen" for one or possibly several 


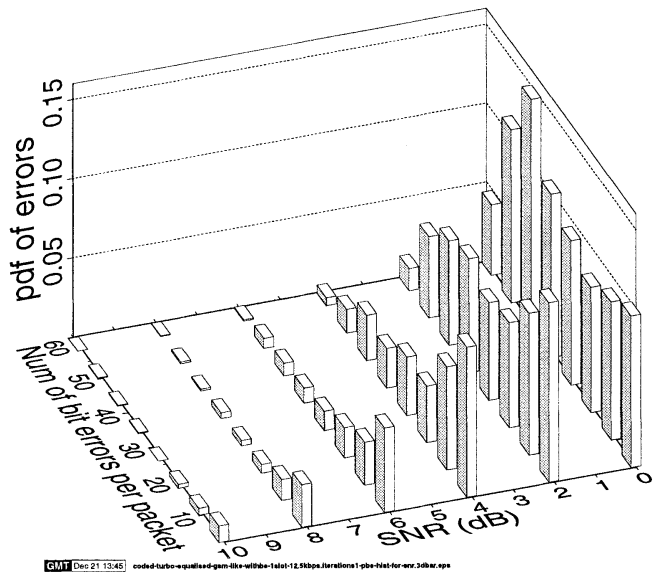

(a)

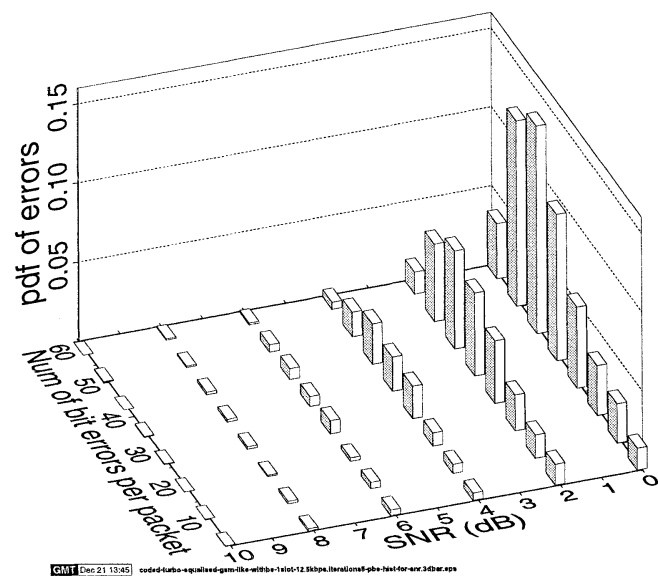

(c)

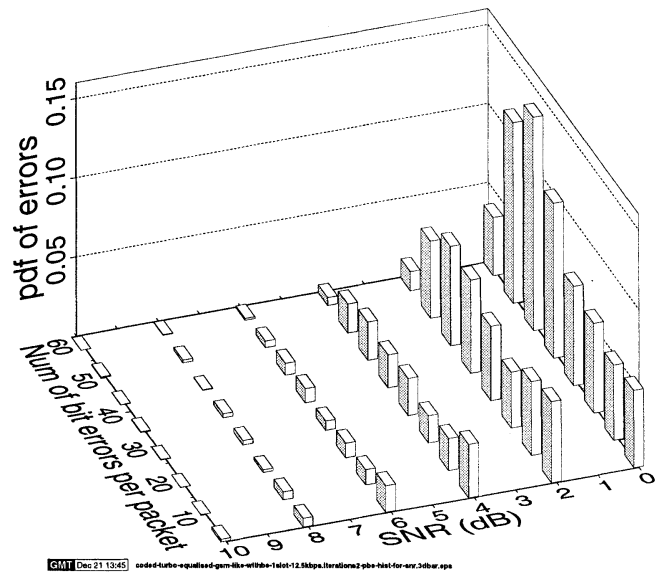

(b)

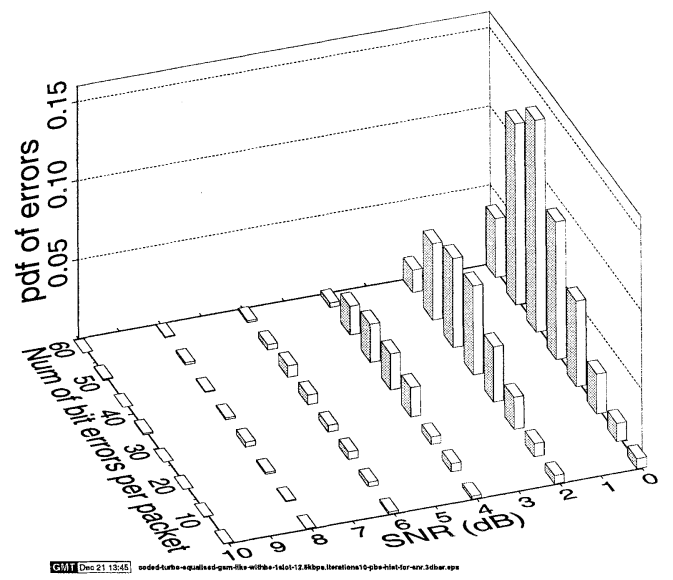

(d)

Fig. 9. PDF of bit errors per 116-bit packet for the one-slot system for a range of channel SNRs and (a) one, (b) two, (c) five, and (d) ten iterations over the channel of Fig. 2.

consecutive video frames. The sudden increases in video quality are achieved, when the system updates the "frozen" part of the video picture in subsequent video frames. The PLR for this scenario was $10 \%$ at the stipulated SNR of $6 \mathrm{~dB}$.

The video quality improved significantly with the aid of two turbo equalizer iterations, while the PLR was reduced from $10 \%$ to $0.7 \%$. In the time interval shown in Fig. 8, there are eight lost video packets, six of which can be seen as sudden reductions in video quality. However, in each case, the video quality recovered with the update of the "frozen" picture areas in the next video frame.

We have found that the maximum acceptable PSNR videoquality degradation with respect to the perfect-channel scenario was about $1 \mathrm{~dB}$, which was associated with near-unimpaired video quality. In Table III, we hence tabulated the corresponding minimum required channel SNRs that the system can operate at for a variety of scenarios, extracted from Fig. 6. As can be seen from the table, the minimum operating channel SNRs for the one- and four-slot system using one iteration is 9 and $7.5 \mathrm{~dB}$, respectively. This corresponds to a system using conventional equalization. A system using two turbo equalization iterations can reduce these operating SNRs to 7.2 and $5.2 \mathrm{~dB}$, respectively. The minimum operating SNRs can be reduced to as low as 5.8 and $3.4 \mathrm{~dB}$ for the one- and four-slot systems, respectively, when invoking ten iterations.

\section{B. Bit-Error Statistics}

In order to demonstrate the benefits of turbo equalization more explicitly, we investigated the mechanism of how turbo equalization reduces the bit error and PLRs. We found that the distribution of the bit errors in video packets after each iteration provided us with interesting insights. Hence, both the PDF and the CDF of the number of bit errors per video packet was evaluated.

Fig. 9 shows the probability density function (PDF) of the number of bit errors in each 116-bit video packet for a range of channel SNRs and for different numbers of iterations. However, the bar for zero bit-errors was removed to aid clarity. Therefore, when more video packets become error-free, the height of the invisible zero bit-error bar increases, and hence the area under the remaining visible bars decreases.

The figure clearly demonstrates that upon each additional iteration the number of erroneous video packets decreases, and-more significantly-the number of packets having a small number of bit errors decreases significantly. However, the probability of packets having a large number of errors, about 40 in this case, is only marginally reduced. Therefore, as expected, turbo equalization can reduce the number of bit errors in packets having a small number of errors, and in some cases reduces the number of errors to zero, thereby improving 


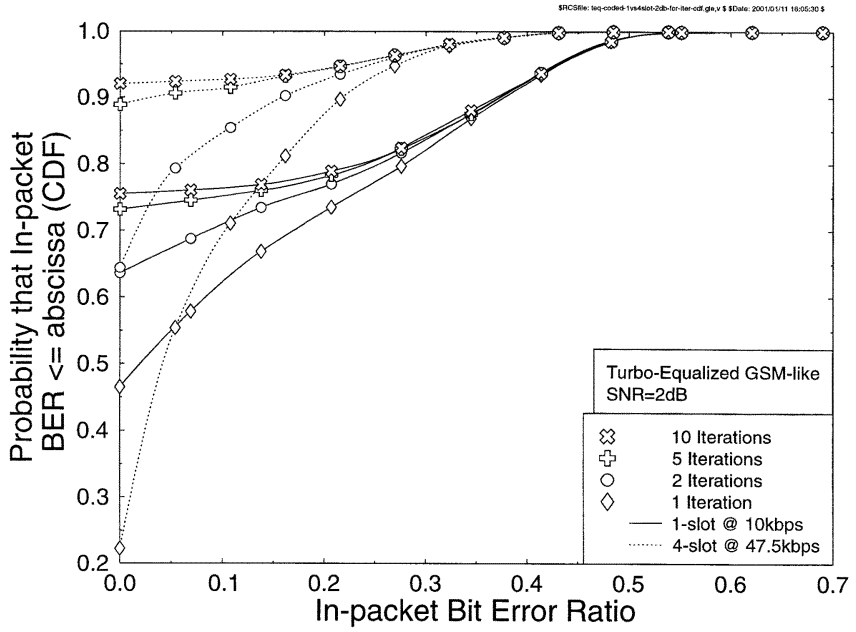

Fig. 10. CDF of the "in-packet" BER at a channel SNR of $2 \mathrm{~dB}$ over the channel of Fig. 2, and for various number of iterations for the turbo-equalized one- and four-slot systems using convolutional coding.

the PLR. However, the packets having a high proportion of bit errors cannot be rendered error-free by turbo equalization.

In order to allow a fair comparison between the one- and four-slot system, we normalized the number of bit errors per packet to the video packet size, hence producing the CDF of "in-packet" BER. Fig. 10 shows the CDF of the "in-packet" BER for a channel SNR of $2 \mathrm{~dB}$ and for one, two, five, and ten iterations, for both the one- and four-slot systems. It should be noted that the value of the CDF for an "in-packet" BER of zero is the probability that a packet is error-free, and hence can be interpreted as the packet success ratio (PSR). The PLR is equal to one minus the PSR. For example, the four-slot system in Fig. 10 at one iteration has a PSR of 0.22 , which corresponds to a PLR of $78 \%$.

Both the one- and four-slot system increase the PSR as the number of iterations increases. For example, the four-slot system increases the PSR from $22 \%$ to $92 \%$, as the number of iterations is increased from one to ten. This corresponds to a reduction in the PLR from 78\% to 8\%. However, the CDF of "in-packet" BER can provide further insight into the system's operation. It can be seen in Fig. 10 that the turbo equalizer iterations reduce the number of packets having "in-packet" BERs of less than $30 \%$. However, the probability of a packet having an "in-packet" BER higher than $35 \%$ is hardly affected by the number of iterations, since the number of bit errors is excessive, hence overwhelming even the powerful turbo equalization.

By referring to Figs. 4 and 5, it can be seen that the four-slot system always has a lower BER, than the one-slot system, although at low SNRs the PLR is higher for the four-slot system. The CDF in Fig. 10 can assist in interpreting this further. Explicitly, the CDF shows that the PSR improves more significantly for the four-slot system, than for the one-slot system, as the number of iterations increases. This is because the four-slot system allows the employment of a longer interleaver, thereby improving the efficiency of the turbo equalizer. However, the $\mathrm{CDF}$ also underlines a reason for the lower BER of the four-slot system across the whole range of SNRs, demonstrating that the

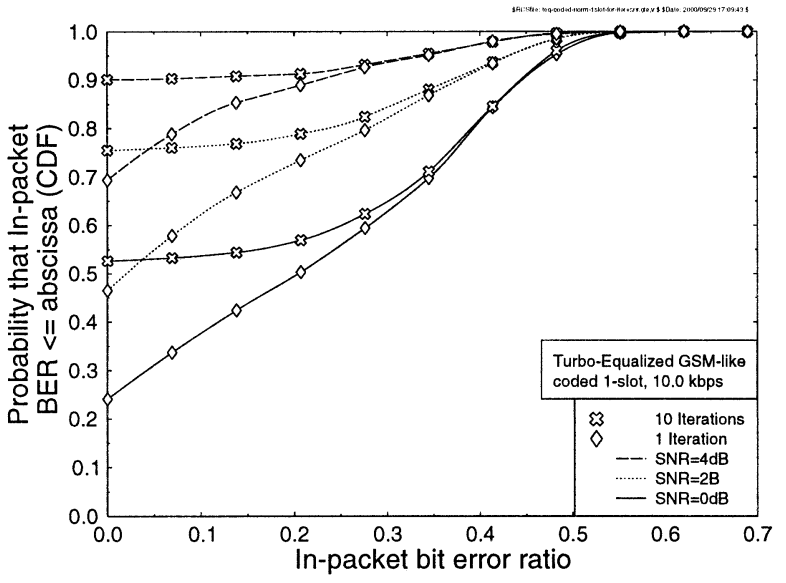

(a)

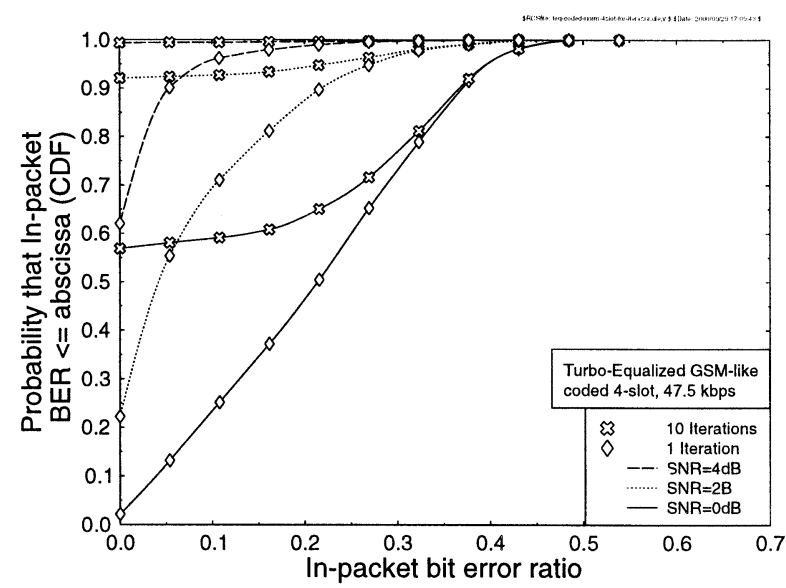

(b)

Fig. 11. CDF of "in-packet" BER performance over the channel of Fig. 2 for the turbo-equalized (a) one- and (b) four-slot system for channel SNRs of 0,2 , and $4 \mathrm{~dB}$ invoking one and ten iterations using convolutional coding.

probability of packets having a high "in-packet" BER is lower for the four-slot system. Since packets having a high "in-packet" BER have a graver effect on the overall BER than those packets having a low "in-packet" BER, this explains the inferior overall BER performance of the one-slot system.

Fig. 11 shows the CDF of "in-packet" BER for conventional equalization and with the aid of ten turbo equalizer iterations for 0-, 2-, and 4-dB channel SNRs. Fig. 11(a) represents a one-slot system, while Fig. 11(b) represents a four-slot system. The figures also show the PLR performance improvement with the aid of turbo equalization.

\section{SUMMARY AND CONCLUSIONS}

The performance of turbo-equalized GSM/GPRS-like videophone transceivers was studied over dispersive fading channels as a function of the number of turbo equalization iterations. Iteration gains in excess of $4 \mathrm{~dB}$ were attained, although the highest per-iteration gain was achieved for iteration indices below five. As expected, the longer the associated interleaver, the better the BER and PLR performance. In Fig. 8, it was observed that the turbo-equalized system was capable of reducing the number of erroneous packets, compared to the conventional noniterative equalization and decoding scheme. Furthermore, Table III 
showed that by using turbo equalization, the operating channel SNR required for achieving a 1-dB PSNR loss was reduced by $3.2 \mathrm{~dB}$ for the one-slot system and by $4.1 \mathrm{~dB}$ for the four-slot system. In conclusion, GPRS/GSM are amaneable to video telephony and turbo equalization is a powerful means of improving the system's performance. Our future work ${ }^{1}$ will improve the system's performance invoking the MPEG4 video codec, using space-time coding and burst-by-burst adaptive turbo equalization.

\section{REFERENCES}

[1] R. Steele and L. Hanzo, Eds., Mobile Radio Communications: Second and Third Generation Cellular and WATM Systems, 2nd ed. New York: Wiley/IEEE Press, 1999.

[2] J. Blogh and L. Hanzo, 3G Systems and Intelligent Networking. New York: Wiley/IEEE Press, 2002. [Online]. Available: http://www-mobile.ecs.soton.ac.uk.

[3] L. Hanzo, P. Cherriman, and J. Streit, Video Compression and Communications Over Wireless Channels: From Second to Third Generation Systems, WLAN's and Beyond. New York: IEEE Press, Mar. 2001. [Online]. Available: http://www-mobile.ecs.soton.ac.uk.

[4] L. Hanzo and J. Streit, "Adaptive low-rate wireless videophone systems," IEEE Trans. Circuits Syst. Video Technol., vol. 5, pp. 305-319, Aug. 1995.

[5] J. Streit and L. Hanzo, "Vector-quantised low-rate cordless videophone systems," IEEE Trans. Circuits Syst. Video Technol., vol. 42, pp. 340-357, May 1997.

[6] - "Quadtree-based reconfigurable cordless videophone systems," IEEE Trans. Circuits Syst. Video Technol., vol. 6, pp. 225-237, Apr. 1996.

[7] P. Cherriman and L. Hanzo, "Programable H.263-based wireless video transceivers for interference-limited environments," IEEE Trans. Circuits Syst. Video Technol., vol. 8, pp. 275-286, June 1998.

[8] L. Hanzo, W. T. Webb, and T. Keller, Single- and Multi-Carrier Quadrature Amplitude Modulation: Principles and Applications for Personal Communications, WATM and Broadcasting. New York: IEEE Press/Wiley, 2000.

[9] L. Hanzo, F. C. A. Somerville, and J. P. Woodard, Voice Compression and Communications: Principles and Applications for Fixed and Wireless Channels. New York: IEEE Press, Aug. 2001. [Online]. Available: http://www-mobile.ecs.soton.ac.uk.

[10] C. Douillard, A. Picart, M. Jézéquel, P. Didier, C. Berrou, and A. Glavieux, "Iterative correction of intersymbol interference: Turbo equalization," Eur. Trans. Commun., vol. 6, pp. 507-511, Sept.-Oct. 1995.

[11] L. Hanzo, T. Liew, and B. Yeap, Turbo Coding, Turbo Equalization and Space-Time Coding. New York: Wiley/IEEE Press, 2002. [Online]. Available: http://www-mobile.ecs.soton.ac.uk.

[12] M. J. Gertsman and J. L. Lodge, "Symbol-by-symbol MAP demodulation of CPM and PSK signals on Rayleigh flat-fading channels," IEEE Trans. Commun., vol. 45, pp. 788-799, July 1997.

[13] G. Bauch, H. Khorram, and J. Hagenauer, "Iterative equalization and decoding in mobile communications systems," in Proc. Eur. Personal Mobile Communications Conf., Bonn, Germany, Sept. 30-Oct. 21997, pp. 301-312.

${ }^{1}$ For information on research in progress and associated publications, please refer to http://www-mobile.ecs.soton.ac.uk.
[14] G. Bauch and V. Franz, "Iterative equalization and decoding for the GSM-system," in Proc. IEEE 48th Vehicular Technology Conf., Ottawa, ON, Canada, May 18-21, 1998, pp. 2262-2266.

[15] _ - "Turbo-detection for enhanced data for GSM evolutionterative equalization," in Proc. IEEE 50th Vehicular Technology Conf., Amsterdam, The Netherlands, Sept. 19-22, 1999, pp. 2954-2958.

[16] "On the application of turbo equalizers in GSM compatible receivers," in Proc. 1998 URSI Int. Symp. Signals, Systems, and Electronics (ISSSE'98), Pisa, Italy, Sept. 29-Oct. 2, 1998, pp. 460-464.

[17] "Study on iterative decoding techniques applied to GSM full-rate channels," in Proc. 1998 9th IEEE Int. Symp. Personal, Indoor and Mobile Radio Communications, Boston, MA, Sept. 8-11, 1998, pp. 1066-1070.

[18] C. Berrou, A. Glavieux, and P. Thitimajshima, "Near Shannon limi error-correcting coding and decoding: Turbo codes," in Proc. Int. Conf. Commun., Geneva, Switzerland, May 23-26, 1993, pp. 1064-1070.

[19] C. Berrou and A. Glavieux, "Near optimum error-correcting coding and decoding: Turbo codes," IEEE Trans. Commun., vol. 44, pp. 1261-1271, Oct. 1996.

Peter Cherriman received the M.Eng. degree in information engineering in 1994 from the University of Southampton, Southampton, U.K., where he is currently working toward the $\mathrm{Ph} . \mathrm{D}$. degree in mobile video networking.

Since 1994, he was with the Department of Electronics and Computer Science, University of Southampton, working on projects for the Mobile Virtual Centre of Excellence. He joined the Research Department of the British Broadcasting Company (BBC), U.K., in 2001. His current areas of research include robust video coding, microcellular radio systems, power control, dynamic channel allocation, and multiple-access protocols.

Bee Leong Yeap received the M.Eng. degree (with first-class honors) in electronics engineering in 1996 and the Ph.D. degree in 2000, both from the University of Southampton, Southampton, U.K.

He has since been continuing his research as a postdoctoral Research Fellow at the University of Southampton. His research interests include turbo coding, turbo equalization, adaptive modulation, and space-time coding.

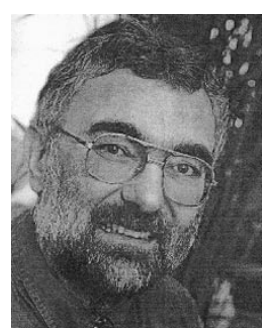

Lajos Hanzo received the M.Eng. degree in electronics in 1976 and the Ph.D. degree in 1983.

During his 26-year career in telecommunications, he has held various research and academic posts in Hungary, Germany, and the U.K. Since 1986, he has been with the Department of Electronics and Computer Science, University of Southampton, Southampton, U.K., where he currently holds the Chair in Telecommunications. He has coauthored eight books on mobile radio communications, published over 400 research papers, organized and chaired conference sessions, presented overview lectures, and has been awarded a number of distinctions. Currently, he is managing a research team, working on a range of research projects in the field of wireless multimedia communications under the auspices of the Engineering and Physical Science Research Council (EPSRC), U.K., the European IST Programme, and the Mobile Virtual Centre of Excellence (VCE). He is a Director of the VCE and also provides a range of industrial training courses.

Dr. Hanzo is an IEEE Distinguished Lecturer. 